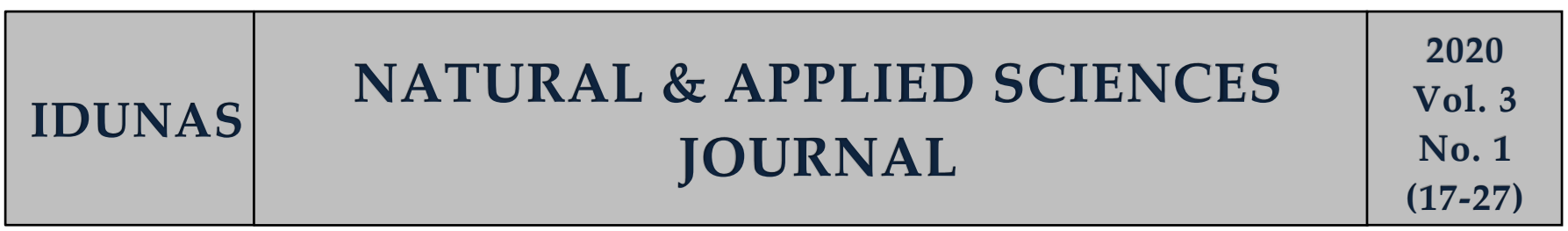

\title{
The Importance of Nano Biosensors and Ethical Elements in Sports Performance Analysis
}

\author{
Review Article \\ Neşe Akpınar Kocakulak ${ }^{1 *(D)}$, Ali Sabahattin Saygın ${ }^{1}$ (iD \\ ${ }^{1}$ İzmır Democracy University, Faculty of Health Sciences, Department of Sport Sciences, IZZMIR/TURKEY \\ Author E-mails \\ nese.kocakulak@idu.edu.tr \\ Tel: +902322601001 \\ Correspondence to: N. Akpınar Kocakulak, İzmır Democracy University, Faculty of Health Sciences, \\ Department of Sport Sciences, İzmir/Turkey \\ DOI: 10.38061/idunas.742366
}

Received: May 25, 2020; Accepted: June 25, 2020

\section{Abstract}

Performance analysis is an indispensable element for elite sports success. Coaches and teams are constantly striving for ways to improve and maximize their performance. Effective use of performance analysis enables tactical, decision making, better athletic confidence and improvement of reflective coaching technique. Performance analysis is required for coaches and athletes to ensure consistent success rates, especially at elite levels. Although performance analysis seems necessary in elite sports, we should recognize the benefits and disadvantages associated with the individual athlete and sports system that regulate their distribution for their use. These are a new technology, nanobiosensor, a concept that combines biomedical field with sports engineering and nanotechnology and ethically addresses future performance analysis in sports. In this study, this concept is discussed. This innovative technology has the potential to revolutionize sports and enables athletes to collect real-time biological data electronically. Affinity-based nanobiosensors have also been taken into consideration in sports medicine and doping control analysis because they are cheap and easy-to-use, yet selectively analytical devices. Allows the use of the same sensor for multiple analyzes. At the same time, nanobiosensors can contribute to filling an important information gap about complementary evidence from their "on-site" use and pre-selecting the risk population of individuals to be targeted for a complete antidoping test; In sports medicine, they can contribute to obtaining analytical knowledge of physiological relevance from measurement of specific parameters or markers before, during and after physical exercise. However, as with many technologies, this technology may have undesirable uses. These unwanted uses are data ownership and privacy may affect data privacy and wellbeing of athletes. While the use of nanobiosensors in sports analysis in the future offers many potential benefits, there is also a concern that they can be abused. For this reason, it is essential for sports organizations to consider the development of a sound, ethically informed governance framework before 
their increased use. Thus, the value of sports and athletes will become more prominent. For this reason, it is very important for athletes and their regulators to develop measures to ethically integrate this technology into sports.

Keywords: Sports, Performance Analysis, Sports Engineering, Nanobiosensors, Ethic

\section{INTRODUCTION}

According to the British Sports Institute, the discipline of performance analysis focuses on improving interventions in the coaching process, allowing performance gains and increasing learning. The discipline of performance analysis stems from the desire to make good performance gains by improving tactics and techniques through feedback. The most important step of the coaching process is to get feedback about the player or team in order to increase the performance of the player and the team (Nic, 2009). Therefore, objective measuring tools are necessary to enable and facilitate the feedback process.Sports Performance Analysis gives coaches objective information that is used to optimize team and player performances. Research has shown that athletes and coaching teams contribute only $30 \%$ of the performance performed by an elite athlete on average, which can only lead to minor gains for the athlete. The remaining 70 percent should be supported by performance analysis (Evans et al., 2017). Performance analysis is not homogeneous. It can be performed according to location, timing, biological and psychological differences. For example, it can take place after the performance of an athlete, or laboratories that provide a more controlled environment. There are several ways in which performance analysis techniques can be used in sports. For example, using visual feedback can be the use of the athlete's profile data to develop a game plan ready to compete successfully in training in a competitive environment. Performance analysts use advanced video performance software systems such as 'Dartfish' to provide this type of information (Dartfish N.D). The increasing sophistication, and reducing cost, of video systems have greatly enhanced post-event feedback, from playback with subjective analysis by a coach to a detailed objective analysis by means of notation systems (Brown and Hughes, 1995). These systems provide athletic advances with advanced analytics that encourage more critical performance feedback and interaction between coaches and athletes.

\subsection{Benefits and Losses of Performance Analysis}

Effective use of performance analysis enables tactical / decision making, improved athletic safety, and improved reflective coaching. Performance analysis is required for coaches and athletes to ensure consistent success rates, especially at elite levels. Performance analysis can be a very useful tool when used to monitor and feedback team skills, strengths, and weaknesses. Coaches or analysts can keep statistics about their team and the opposing team. He can make comparisons as to which side does what better and why he performs better. Performance analysis can also be a very useful tool when used to monitor individual players' skills, and their ability to carry out game-plans and strategies. Stats can be produced to point out the player's strengths and weaknesses, which is essential when optimizing performances (Taylor, 2017). Although performance analysis may seem necessary in elite sports, we should recognize the advantages and disadvantages associated with the individual athletes and sports systems that regulate their distribution for their use (Table. 1). 
Table 1. Advantages and disadvantages to both the athlete and the sports system

\begin{tabular}{|l|l|l|l|}
\hline $\begin{array}{l}\text { Advantages to the } \\
\text { Athlete }\end{array}$ & $\begin{array}{l}\text { Advantages to the } \\
\text { System }\end{array}$ & $\begin{array}{l}\text { Disadvantages to the } \\
\text { Athlete }\end{array}$ & $\begin{array}{l}\text { Disadvantages to the } \\
\text { System }\end{array}$ \\
\hline $\begin{array}{l}\text { Performance data } \\
\text { increases. }\end{array}$ & $\begin{array}{l}\text { It provides, safety in } \\
\text { sports }\end{array}$ & $\begin{array}{l}\text { It harms athlete's } \\
\text { consent, } \\
\text { confidentiality and } \\
\text { data ownership. }\end{array}$ & $\begin{array}{l}\text { With technology } \\
\text { doping, it creates } \\
\text { unfair competition. }\end{array}$ \\
\hline $\begin{array}{l}\text { It creates added value } \\
\text { for the athlete. }\end{array}$ & $\begin{array}{l}\text { It discourages } \\
\text { cheating. }\end{array}$ & $\begin{array}{l}\text { It makes the athlete } \\
\text { unskilled. }\end{array}$ & $\begin{array}{l}\text { Facilitates corruption } \\
\text { / decay. }\end{array}$ \\
\hline
\end{tabular}

Recent advances in bioengineering are essential for performance analysis. The development of sensor and image processing and feedback systems enables analysis by transferring data in a digital environment. This has enabled much more effective feedback systems to be developed with increased data levels to be achieved in less time (Baca et al., 2006). If we look at how performance analysis and feedback systems are used in swimming sports, swimmers of the Great Britain National Team can be an example of this system. The way the system works is to follow a swimmer from the water wirelessly (Epsrc, 2012) to ensure that all movements of the athlete (such as body position, acceleration, and general technique) are tracked. The information obtained is transmitted to analysts and coaches who can provide feedback to make real-time adjustments during training. Then the coaching team should provide feedback to swimmers through observers and post-video training, but this can sometimes result in loss of precision and time. Despite these benefits of the system, it is limited as it only focuses on the external movements of an athlete. For instance, to learn about the relative fatigue of athletes, should be given a lactate test and blood samples and such data should be analyzed far from the field of training as should be taken in mid-training. As a result, sports engineers have explored other areas of research such as biomedical and engineering to solve this problem. As a solution, they developed biosensors to capture biological data via mobile devices. Some sports models use wearable technology to calculate and record basic physiological data and instant performance data. (Smith, I. A. 2014). Biosensors can coach athletes and wirelessly collect performance data such as athlete's acceleration position, respiratory rates, and fatigue levels. It allows continuous monitoring of an athlete (Moskvitch, K. 2012). In this way, the physical condition of the athlete can be followed more extensively and gives more information about how the body parts look like during training and competition. Also, data collected with biosensors can be used to predict what could put an athlete's health at risk, thereby promoting early diagnosis of conditions such as cardiac arrest, making the sport potentially safer for the athlete (Moskvitch, K. 2012). In summary, we can say that the health profiles of biosensors and athletes can be monitored completely. However, we should ask more precisely which biosensors are and how they can be applied in elite sports.

\subsection{Feedback Systems: Biosensors / Nanobiosensors Performance Analysis Systems}

Before dealing with the ethical aspect of nanobiosensors, we should think about what the biosensor is. It describes the Higson biosensor as "a chemical sensing device in which the presence of a biologically derived recognition is connected to a transformer to enable numerical development of some complex biochemical parameters" (Higson et al., 1994). Fraser, on the other hand, defines it as an "analytical device 
containing a deliberate and intimate combination of specific biological elements and physical elements" (Fraser., 1994). Biosensors consist of a bio element and a sensing element (Mohanty et al., 2006). Using a biosensor, an athlete's performance can be maximized because it can keep athletes at their highest physical performance levels. However, it is very difficult to stay in this state when exercising at the extreme for a long time. It is often said that the health condition is good where the elite condition starts. Therefore, precise, accurate and timely monitoring is very important for athlete-centered sports technology and sports medicine (Dijkstra et al., 2014). An example of a biosensor used for sports is a Pulse oximeter (Sheehan, K. 2010). This is a hand-held electronic device used to measure the amount of oxygen in the blood of athletes. It emits infrared light by plugging and running on an athlete's forefinger. Oxygenated blood absorbs the blood of athletes at different levels, which allows the calculation of the correct oxygen level (Montgomery, 2010). Pulse Oximeter is used to monitor potential oxygen drops during such situations and allows coaches and athletes to develop new methods to increase endurance for higher competitive gains. Given the change in the lactate concentration in the body fluids of the athletes during physical activity, the lactate test is a test that determines the condition and endurance capacity of the athlete during the exercise by monitoring the lactic acid level. The most commonly used method for determining the form of individuals and determining individual training weight is the lactate test. The barcode system obtained to make measurements during exercise is integrated into the adhesive tape, sportswear, sweatbands for the waist and head as seen in Figure 1 (Martinez, A.W. 2011).

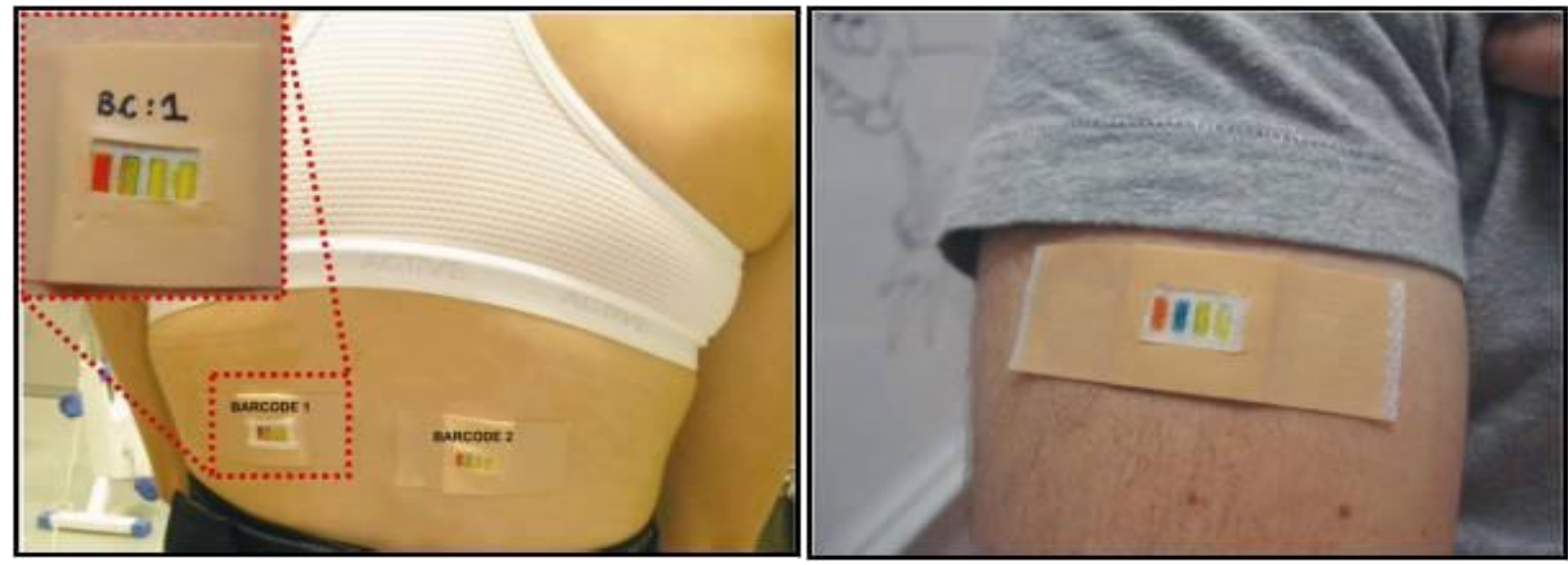

Figure 1. Barcode systems on the athlete's back and arm

The use of such sensors for sweat analysis provides valuable physiological information for applications in sports performance (Coyle et al., 2009). RBC Life Sciences ${ }^{\circledR}$, Inc. The "Nanoceuticals TM Slim Shake Chocolate" produced by the product contains nanoparticles that clean free radicals more effectively, increases hydration, regulates body $\mathrm{pH}$, reduces the amount of lactic acid formed during sports, and reduces the surface tension (www.nanotechproject.org). However, the volume of biological data that biosensors (1) can collect; (2) practical functionality in terms of size and body restriction for elite athletes; and (3) they have extreme sensitivity, which can sometimes lead to incorrect data measurement. As a result, sports and biomedical engineers tried to overcome these deficiencies with nanobiosensors. Nanobiosensors generally differ from biosensors only in scale. A nano biosensor can be defined as a biosensor consisting of nanomaterials and dimensions on the nanometer (Atta et al., 2011). The incredibly small nanobiosensors have many advantages due to the large surface area/volume ratio. Many nanomaterial atoms are located 
near their surface, which leads to improved transduction and signal capabilities (Malik et al., 2013). This increases their ability to detect and provide more accurate data logging. Also, these devices can be installed on a person for a long time without irritation.

\subsection{Analysis of Performance Feedback Systems as an Example of Nanobiosensors}

Nanobiosensors offer enormous potential for elite athletes and are at the stage of research, with teams around the world developing and trying new ways to use them in the elite world of sports and medicine. Nanotechnology engineers have developed a Nanotattoo that can be placed on an athlete's arm to monitor lactate sweating levels (Jia et al., 2013). Lactate monitoring is an important indicator for evaluating physical performance during training and competition with multiple sprints and speed resistance. Traditionally, lactate levels have been monitored using lactate sensor strips used with a hand-held device that is both convenient and uncomfortable during physical activity (Jia et al., 2013). In contrast, the non-invasive enzymatic temporary transfer tattoo acts as a flexible sensor made by screen printing methods bent to fit the body. Determining more precise measures of athlete lactate levels, which is a significant performance barrier, allows significant improvements for athletes and coaches; because it enables the immediate acquisition of data on biological performance, allowing immediate intervention in training sessions. Let's look at the advantages of some nano biosensors and biomarkers. Corner technique is used to evaluate the training data of boxers. Thanks to the three-axis accelerometer on the produced glove, it is possible to measure the characteristics of athletes such as stroke forces, stroke combinations, stroke rates, and blocking times. This ensures that the data of the athlete is stored during training and the performance of the athletes is improved. Goal post-integration using sensor fusion has recently been implemented on two technical bases to scan a specific area. The first one is based on the cameras installed in the stadium structure, and at the same time, it makes a decision based on the position of the ball relative to the target line based on the view of at least three different cameras simultaneously. The second technique is based on magnetic field sensors placed on three goalposts, where the decision will be made based on the magnetic field change. Both techniques process the signals from the sensors and transmit them to the referees via wireless encrypted communication (Shan, P., 2014). To investigate the physical performance of an athlete on the field, a GPS (Global Positioning System) is used to collect data such as speed, position, acceleration, time of each activity type in each player. Therefore, the use of sensor fusion for football analysis is essential for rule enforcement and monitoring and evaluation of players (Kocakulak, 2019). Another sensor application is designed for athletes struggling in basketball and baseball, and the interactive armband can be used to determine the beat and shot rhythms of the athlete and to create a muscle rhythm map. The proposed basketball instruments were developed using nine accelerometers placed on the ball that communicate with mobile devices and allow the user to receive data through an application installed on the device. This type of analysis helps correct movement and shots to improve the quality and accuracy of field targets (Kocakulak, 2019). "Readiband" electronic tapes designed for athletes can also measure how much sleepers sleep at night and sleep quality. Thanks to the accelerometer in the band, it detects whether the athlete is awake by measuring the movements of her wrist. hanks to the SAFTE (Sleep, Activity, Fatigue, Task, and Activity) module, a sleep quality score indicating the sleep quality of the person is calculated.

Let's look at some ethical and legal concerns before nano biosensors are widely applied. 


\subsection{Benefits to the Athlete}

Higher Value Performance Data:

Previously, performance analysis was limited due to the inability to prepare biological data for analysis. However, with the integration of biosensors and nanobiosensors, coaches and athletes began to better understand how an athlete's body works. Thanks to the future use of nanobiosensors in the form of wearable technology, a range of bodily functions, dehydration, healing, lactate levels, and even wound healing rates can be tracked wirelessly will present the picture (Ray et al., 2019). In addition, nanobiosensors increase the gather speed and usability of the data, providing emergency interventions to coaches and athletes with data transmitted in real time on their smartphone or tablet. Wearable microfluids touch the human skin directly with wearable technology, so that data can be analyzed by wireless data transfer (Kocakulak et al., 2019).

If we exemplify the performance analysis data measurement with the football branch; GPSPORTS training vest technology is also widely preferred for team sports today, while it is a smart sensor especially used in football branch. Arrangement of training data and stations can be made with the vest design that allows the measurement of the movements, body activity and performance data of the athletes in the team (Akçalı., 2016).

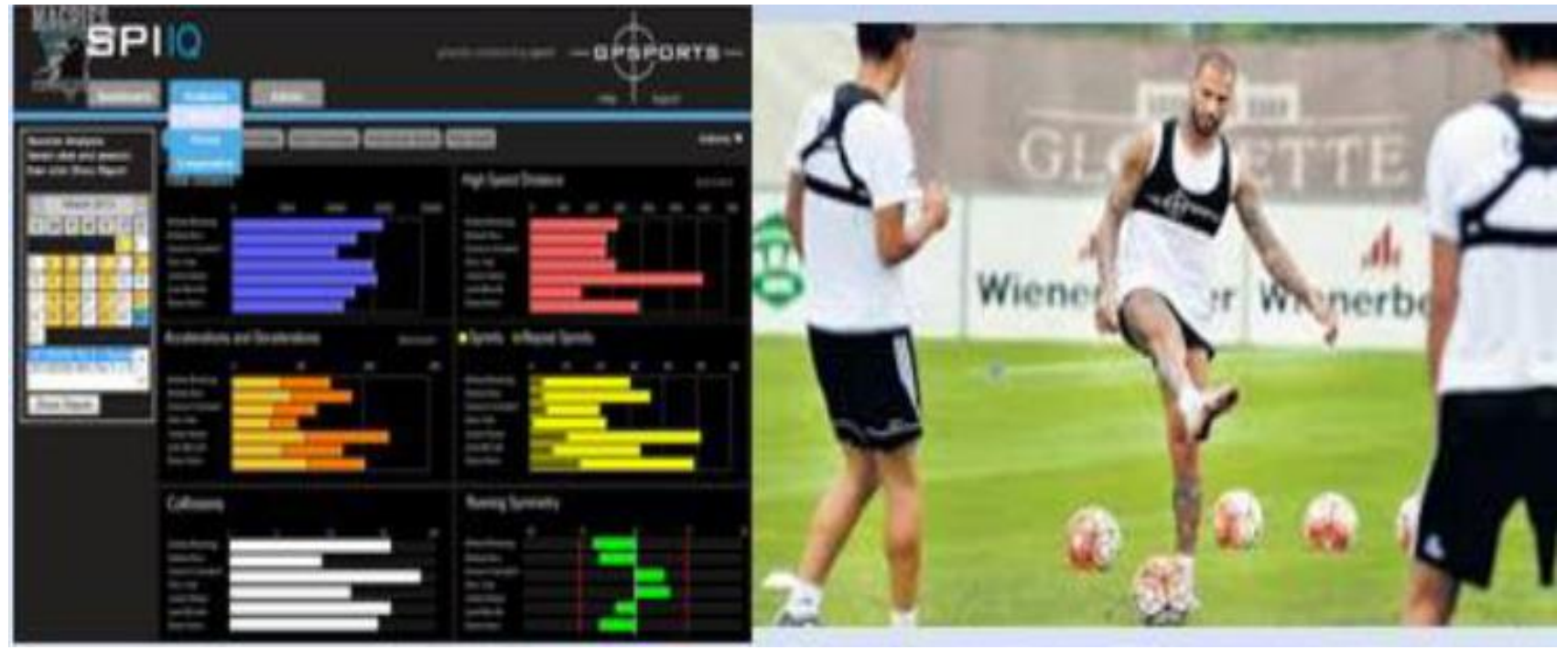

Figure 2. GP SPORTS training vest technology (Akçalı. 2016).

Increased value for the athlete:

In today's modern sports world, statistics based on the performance of the players during the season have become very important for economic contract negotiations. High-performance data can provide contract opportunities for athletes and coaches. Recent developments in biosensors have provided significant improvements in the study of athletes' biological data, along with indicators of future injury rates for athletes in the long term. Strong biometric data positively affect an athlete and club. It also increases the economic value of the player as well as the success and economy of the club. Other measures focus on the lactate production level, heartbeat, but nano biosensor is used to further justify data readings. It can make 
direct comparisons between players' biological data. This can also be advantageous for clubs, as it can provide coaches and managers with data that can rely on depositing quality players without fear of return due to long-term injury.

\subsection{Disadvantages of the Athlete}

The qualification of the athlete:

The more general use of engineering in sports can render athletes unskilled, more confident in the technology (Hunter., 2011; Miah., 2006). The athlete does not want to become unskilled. The use of technological innovation has made performance comparisons of many athletes less meaningful. The role of sports engineering is to support the development of an athlete. Therefore, it is essential to use nanobiosensors to support the athlete rather than eliminating the skill level required to become an elite athlete (Carr., 2008).

\section{Consent, Privacy and Data Ownership:}

The current use of biosensors and the use of future nanobiosensors for performance raises serious concerns surrounding the collection of biological data. Despite the benefits that nanobiosensors may bring to the performance of an athlete in the future, it should be carefully balanced against key issues such as privacy, security, consent, and data ownership (Meingast et al., 2006). The use of nanobiosensors in sports requires clear and controlled regulation. Even doctors take the consent of his/her patient and tell him about the risks of what he/she will do before taking any action (Kegley., 2004). Also, the data of athletes using this nano can pass to third parties at once (Meingast et al., 2006). The use and storage of data that could harm the interests of individual athletes is an issue that needs to be addressed ethically. The questions of who owns such data and who owns related information flows are important. There are serious concerns about privacy and security regarding the use of nanobiosensors. The data collected and stored by the athletes can be captured by hacking, and this can give other teams an advantage. Also, for example, the physiological life signals of athletes are sensitive and can determine that they have an embarrassing illness or a careerending situation, so leaking this information undermines the privacy (Kumar et al., 2012). The constant use of nanobiosensors raises concern about the blurring of the private / public distinction. 24/7 monitoring of athletes again undermines the privacy of private life.

\subsection{Benefits to the Sports System}

Improves Safety in Sports:

Sports engineering increases safety in sports. This can be exemplified by the development of wearable items, from clothing to shoes. The growth of nanobiosensors in the future may provide more safety in sports. The use of biosensors can reveal a safer playground, help prevent injuries, ensure that athletes comply with vital health data to risk standards (Evans et al., 2017). With the use of such biosensors, injuries and increased sensitivity are prevented by creating a safer playground. It can be used to control vital health data and ensures that athletes comply with agreed risk standards. 
During the match played with the developed Checklight technique, the severity of the pulses taken is measured in order to control the blows to the head area and to protect the athlete's health. This product can be measured in the sports branches (ice hockey, American football), where the physical contact is intensely carried out, and the severity of the blows received with a helmet placed inside the helmet. Thus, the first intervention is performed healthier and the health of the athletes can be protected according to the severity of the blow received (Akçalı., 2016).

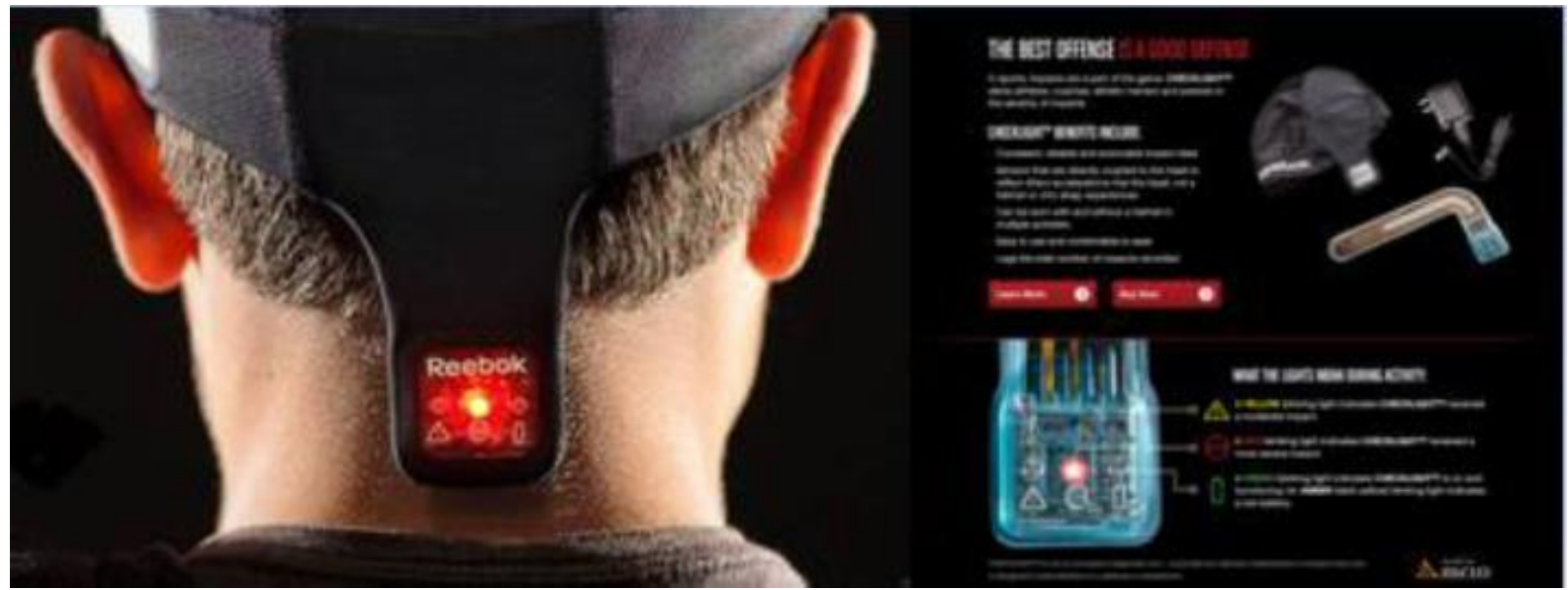

Figure 3. Check light technology product example (Akçalı., 2016).

Therefore, this new technology is not only limited to the performance analysis required in sports, it is also very important for the athlete's safety in order to prevent long-term injuries.

Prevents cheating:

In the current system, some drugs can be saved from doping tests, that is, they are not caught. Moreover, the application of micro doping made it extremely difficult to detect doping. Increasing the development and use of biosensors and nanobiosensors can bring a better application with regulator balance thanks to increased sensitivity to cheating. Biosensors can be integrated into wearable microfluidic chips to meet different requirements of testing body values with fewer technical barriers (Yi-Qiang et al., 2017). With the Athletes' Biological Passport, the athletes can be tracked even while on holiday and checked whether they cheat (MacGregor et al., 2013). Nanobiosensors can be used to further develop this feature (continuous monitoring) due to greater sensitivity and more real-time tracking potential. This can be very useful in increasing confidence in sports, especially in sports such as athletics and cycling, where doping scandals undermine sports' reputation and commercial value.

\subsection{Disadvantages of the Sporting System}

Unfair Competition in Sport: “Technology Doping":

Technology doping is a new concept. It refers to giving an athlete an advantage by using technology. After all, sports are a competitive business and, for example, takes the lead if the British cycling team can produce better bicycles than in other countries. Or financial fair play aims to balance the spending on 
football teams and avoid unfair competition. Concerning nanobiosensors, the issue of technology in justice and sports can also be brought up. We have mentioned that the technology offers athletes to access higher levels of biological data for performance analysis. We can reasonably assume that not all athletes can access these sensors due to the high patent costs; It is an unfair advantage for those who have those sensors. For example, during a marathon or bike race, athletes can use Nanobiofeedback to correct their tactics. The coaching team may notice, for example, that the athlete's glucose level is decreasing, and therefore advise the athlete to take glucose as soon as possible to maintain consistent energy levels that can determine winning or to lose. Therefore, deciding when to run a marathon runner, thanks to nano biosensors, potentially creates unfair competition. There is also a concern that sports can be a test of the power of technology systems, not a test of athletes' abilities.

\section{Corruption / corruption:}

Using nano biosensors for performance management provides many potential benefits, as mentioned earlier, but their use in elite sports also has the potential to increase corruption in sport. Providing real-time transmission of the biological data of an athlete or team equipped with nano biosensors with WI-FI in the future may result in exposure to data piracy by deliberate theft or leakage of important information to competitors. If doping is the biggest threat to the integrity of the sport, match tuning and handicapping is the second. We can count the good properties of nano's, but they can also bring corruption in sport. It is quite possible scenarios that the data of the team and players are stolen or that someone inside leaks to the opponent team. Spending on this way, the opposing team gains knowledge of the opponent's tactics and all kinds of in-field situations of key players. Stealing biological data is also effective in the match setting. While it is ensured that nano biosensors are added to the elite sports, it is very important that the relevant institutions meet the potential losses that may arise due to this technology.

\section{CURRENT AND POTENTIAL SOLUTIONS TO ETHICAL PROBLEMS OF} NANOBIOSENSOS IN SPORTS

An athlete may sign legal documents to ensure that their biological data is protected from abuse, properly stored, and not used against them. There are regulatory institutions on such issues related to consent. One of the most important future developments in the integration of nanosensors is the development of a regulatory framework and policy for their use in sports. A regulation set will protect both the athlete and the system against contamination. Although regulators exist to protect an athlete's data and privacy, concerns remain about privacy and consent. It is therefore important that sports organizers work together to develop a governance framework that can be applied when preparing for this technology. A regulatory framework will be required that sets limits on how these sensors are used in athletes' private lives. We can predict the 7/24 data collection cycle to take advantage of the nano biosensor potential. This will increase even more during peak competition times but will collect performance data that is not important even at rest. Also, a well-planned security network must be created and used to protect the wireless network where nano biosensors will work without potential hacking, and to protect the biological data of athletes, to ensure privacy and confidentiality (Kumar et al., 2012). This will allow athletes to have more confidence in using nano biosensors and therefore will be more willing to use. Consequently, these are key elements that need to be discovered and developed before their integration into elite sports and to 
ensure that all future concerns are fully evaluated. It is essential to take precautionary measures at any intermediate stage to promote the safe and ethical use of nanosensors for both athlete's well-being and the elite sports system as a whole. This will also help raise the ethical profile of technological development in sports, and thus will be seen as an essential element of sports management. Despite all these data, nanosensors are not yet used in a wide range of sports. Because it is a very expensive and difficult technology, it cannot be used by everyone. According to some sports circles, this situation creates unfair competition. For example, the International Federation of Swimming (FINA) banned nanotechnologysupported swimsuits as "technology doping" in 2010 on the grounds that it created unfair competition. Regardless of the sports branch realized in such cases, athletes or teams that are stronger in monetary terms are thought to gain an advantage to their rivals thanks to this technology. Athletes with limited abilities are thought to perform better with the help of nanotechnology products (Kocakulak, 2019; MacGregor, 2013). In order to eliminate this negative situation, it may be an alternative solution to pull the nano-supported products to the levels that everyone can reach in the field of sports and thus prevent unfair competition that may occur.

\section{CONCLUSIONS}

Performance analysis is an important training and competition tool for every elite athlete and ensures that they continue to achieve basic marginal gains through reflective feedback practices. Performance analysis is also a constantly evolving practice that includes areas such as sports engineering and biomedical research, which use the latest technology to get more analysis and feedback from athletes. The integration of biosensors in sports performance analysis has enabled the acquisition of biological data for new levels of feedback about the performance of athletes in both training and competition. This integration has begun to provide significant benefits to athletes in the world of sports, and with the future developments of nanobiosensors, all of these are likely to improve further. However, before nanobiosensors become a daily tool in sport performance analysis and development, regulatory agencies should be taken into account in order to take into account the points in which access to data is not taken into account - data access, ownership, privacy, privacy as well as athlete well-being. Thus, the value of sports and athletes will become more prominent. It is therefore important to develop measures for sports organizations and regulators to ethically integrate this technology into sports.

\section{REFERENCES}

1- Evans, R., McNamee, M., Guy, O. (2017). Ethics, Nanobiosensors and Elite Sport: The Need for a New Governance Framework. Sci Eng Ethics (2017) 23:1487-1505

2- Baca, A., Kornfeind, P. (2006). Rapid feedback systems for elite sports training. Pervasive Computing IEEE, 5(4), 70-76.
3- Higson, S. P. J., Reddy, S.M., Wadgama, P. M. (1994). Enzyme and other biosensors: Evolution of a technology. Engineering Science and Education Journal, 3(1), 41-48.

4- Fraser, D. M. (1994). Glucose biosensors-The sweet smell of success. Medical Device Technology, 5(9), 44-47. 
5- Mohanty, S. P., Kougianos, E. (2006). Biosensors: A tutorial review. Journal of IEEE Potentials, 25(2), 35-40.

6- Dijkstra, H. P., Pollock, N., Chakraverty, R., Alonso, J. M. (2014). Managing the health of the elite athlete: A new integrated performance health management and coaching model. British Journal Sports Medicine, 48(7), 523-531.

7- Atta, N.F., Ahmed, G., Shimaa, A. (2011). Nanobiosensors for health care, Biosensors for health, environment and biosecurity. http://www.intechopen.com/books/biosensors-forhealth-environment-and biosecurity/nanobiosensors-for-health-care. Accessed April 15, 2016.

8- Malik, P., Katyal, V., Malik, V., Asatkar, A., Inwati, G., Mukherjee, T. K. (2013).

Nanobiosensors: Concepts and variations. ISRN Nanomaterial, 2013, 1-9.

9- Jia, W., Bandodkar, A., Ramirez, G., Windmiller, J., Yang, Z., Ramirez, J., et al. (2013). Electrochemical tattoo biosensor for real-Time noninvasive lactate monitoring in human perspiration. Analytical Chemistry, 85(14), 6555366560.

10- Akpınar, K, N., Ünal, İ. (2019). Biochips for Physical Exercise Studies. Natural \& Applied Sciences Journal, Vol. II, No. 1,16-27.

11- Akçalı, K. (2016). Farklı Spor Branşlarında Kullanılan Akıllı Tekstil Ürünlerinin İncelenmesi. International Journal of Science Culture and Sport December 2016: 4 (Special Issue 3)

12- Yi-Qiang, F. A. N., Feng, G. A. O., Mei, W., Zhuang, J., Gang, T., Zhang, Y. J. (2017). Recent development ofwearable microfluidics applied in body fluid testing and drug delivery. Chinese Journal of Analytical Chemistry, 45(3), 455-463.

13- MacGregor, O., Griffith, R., Ruggiu, D., McNamee, M. (2013). Anti-doping, purported rights to privacy and WADA's whereabouts requirements: A legal analysis. Fair Play: Revista de Filosofía, Ética y Derecho del Deporte, 1(2), 133.

14- Kumar, P., Lee, J. H. (2012). Security issues in healthcare applications using wireless medical sensor networks: A survey. Journal of Sensors (Basel), 12(1), 55-91. 